3. E. Egervary, On the smallest convex cover, Publ. Math. Debrecen vol. 1 (1949) pp. 65-70.

4. T. Bonnesen and W. Fenchel, Theorie der konvexen Koerper, Ergebnisse der Math. vol. 1, 1934.

5. G. A. Bliss, Lectures on the calculus of variations, University of Chicago Press, 1946.

6. E. A. Coddington and N. Levinson, Theory of ordinary differential equations, McGraw-Hill, 1955.

7. W. Blaschke, Kreis und Kugel, Chelsea, 1949.

MCGill University

\title{
EXTENDING DIFFEOMORPHISMS
}

RICHARD S. PALAIS

In [ 1 , Theorem 5.5], the author proved the following fact. Let $M$ be a differentiable manifold, $p \in M$, and $f$ a diffeomorphism of a neighborhood of $p$ into $M$. If $M$ is orientable assume in addition that $f$ is orientation preserving. Then there exists a diffeomorphism of $M$ onto itself which agrees with $f$ in a neighborhood of $p$. In this paper we shall answer affirmatively a question raised by A. M. Gleason; namely whether the 0 -cell $p$ can be replaced by a differentiable $k$-cell. It turns out that this extension follows rather easily from the special case. The author understands that the theorem has been proved independently by J. Cerf in his thesis (not yet published). The author would like to thank Dr. S. Smale for several suggestions utilized in this paper.

1. Notation. $M$ will denote an $n$-dimensional differentiable $\left(=C^{\infty}\right)$ manifold. For $t=\left(t_{1}, \cdots, t_{k}\right) \in R^{k}$ we write $\|t\|^{2}=\sum_{i=1}^{k} t_{i}^{2}$. We put $B_{r}^{k}=\left\{t \in R^{k} \mid\|t\| \leqq r\right\}$ and $\dot{B}_{r}^{k}$ for the interior of $B_{r}^{k}$ in $R^{k}$. We regard $B_{r}^{k}$ as a subset of $B_{r}^{k+l}$ by $\left(t_{1}, \cdots, t_{k}\right) \rightarrow\left(t_{1}, \cdots, t_{k}, 0 \cdots 0\right)$. By a differentiable $k$-cell in $M$ we mean a one-to-one map $\phi: B_{1}^{k} \rightarrow M$ such that for each $t \in B_{1}^{k}$ there is a neighborhood $U$ of $t$ in $R^{k}$ and a nonsingular differentiable map $f: U \rightarrow M$ such that $f$ and $\phi$ agree on $U \cap B_{1}^{k}$. It follows from well-known extension theorems that if $\epsilon>0$ is sufficiently small then $\phi$ can be extended to a one-to-one nonsingular differentiable map of $\dot{B}_{1+\epsilon}^{k}$ into $M$. We will write $|\phi|$ for the image of $\phi$. We note that a differentiable $n$-cell $\phi$ in $M$ determines a coordinate system in $|\phi|$, and hence an orientation of $M$ if $M$ is orientable.

2. Extending a $k$-cell to an $n$-cell. The theorem of this section ex-

Received by the editors June 3, 1959. 
presses what is probably a quite well-known fact. However the author knows of no reference. The idea of the present proof was suggested by S. Smale.

THEOREM A. If $\phi$ is a differentiable $k$-cell in $M$ and $U$ is a neighborhood of $|\phi|$ then there exists a differentiable $n$-cell $\psi$ in $M$ with $|\psi| \subseteq U$ and $\phi=\psi \mid B_{1}^{k}$. If $k<n$ and $M$ is orientable we can assume that $\psi$ defines either orientation of $M$.

Proof. Without loss of generality we can assume that $U=M$ and that $M$ is paracompact and hence admits a Riemannian metric which we now fix. Let $\phi^{\prime}: \dot{B}_{1+\epsilon}^{k} \rightarrow M$ be a one-to-one, nonsingular differentiable extension of $\phi$ and let $\Sigma$ be the image of $\phi^{\prime}$, a $k$-dimensional submanifold of $M$. Let $\Sigma^{\prime}=\phi^{\prime}\left(\dot{B}_{1+\epsilon / 2}^{k}\right)$ and let $N(\delta)$ be the part of the normal bundle to $\Sigma$ lying over $\Sigma^{\prime}$ and consisting of vectors of norm $\leqq \delta$. Since $\Sigma^{\prime}$ is relatively compact in $\Sigma$ it follows that $E$, the restriction of the exponential map of $M$ to $N(\delta)$, is a diffeomorphism of $N(\delta)$ onto a neighborhood of $|\phi|$ in $M$ if $\delta$ is sufficiently small. By a change of scale in the metric we can suppose this is so for $\delta=2$. Since $\Sigma^{\prime}$ is contractible $N(2)$ is equivalent to the product bundle, i.e. we can find a diffeomorphism $T$ of $\Sigma^{\prime} \times B_{2}^{n-k}$ onto $N(2)$ which maps $x \times B_{2}^{n-k}$ linearly and orthogonally onto the set of vectors of norm less than or equal to two which are orthogonal to $\Sigma^{\prime}$ at $x$. We now define $\psi: B_{1}^{n} \rightarrow M$ by

$$
\psi\left(t_{1}, \cdots, t_{n}\right)=E\left(T\left(\phi\left(t_{1}, \cdots, t_{k}\right), t_{k+1}, \cdots t_{n}\right)\right) .
$$

It is clear that $\psi$ can be extended to a diffeomorphism of $B_{1+\epsilon / 2}^{n} \rightarrow M$. If $t_{k+1}=\cdots=t_{n}=0$ then $T\left(\phi\left(t_{1}, \cdots, t_{k}\right), t_{k+1}, \cdots, t_{n}\right)=$ zero vector at $\phi\left(t_{1}, \cdots, t_{k}\right)$ so $\psi\left(t_{1}, \cdots, t_{n}\right)=\phi\left(t_{1}, \cdots, t_{k}\right)$, i.e. $\psi \mid B_{1}^{k}=\phi$. The final statement of the theorem is clear, for if $\psi$ defines one orientation of $M$ then $\left(t_{1}, \cdots, t_{n}\right) \rightarrow \psi\left(t_{1}, \cdots, t_{n-1},-t_{n}\right)$ defines the other, and has all the other required properties. q.e.d.

\section{Transitivity of diffeomorphisms on $k$-cells.}

Theorem B. Let $\phi$ and $\psi$ be two differentiable $k$-cells in $M$. If $k=n$ and $M$ is orientable assume in addition that $\phi$ and $\psi$ define the same orientation of $M$. Then there exists a diffeomorphism $F$ of $M$ onto itself such that $\psi=F \circ \phi{ }^{1}$

${ }^{1}$ Recall that the sum of two $n$-manifolds $M_{1}$ and $M_{2}$ is defined by taking differentiable $n$-cells $\psi_{1}$ and $\psi_{2}$ in $M_{1}$ and $M_{2}$ respectively and identifying $M_{1}-\psi_{1}\left(\dot{B}_{1}\right)$ and $M_{2}-\psi_{2}\left(\dot{B}_{2}\right)$ along the boundaries of $\left|\psi_{1}\right|$ and $\left|\psi_{2}\right|$ in the obvious way. As B. Mazur has pointed out to us, it is precisely Theorem B which is needed to show that the sum (to within diffeomorphism) is independent of the choice of $\psi_{1}$ and $\psi_{2}$. Because of Corollary 1 this extends to the sum of manifolds with boundary. 
Proof. By Theorem A we can assume that $k=n$. The map $\phi(t) \rightarrow \psi(t)$ defines a diffeomorphism of $\phi\left(\dot{B}_{1}^{n}\right)$ onto $\psi\left(\dot{B}_{1}^{n}\right)$ which is orientation preserving if $M$ is orientable. By Theorem 5.5 of [1] if $\epsilon$ is sufficiently small and positive we can find a diffeomorphism $H$ of $M$ onto itself such that $\psi(t)=H(\phi(t))$ if $t \in B_{\epsilon}^{n}$. Let $\lambda$ be a monotone nonincreasing differentiable real valued function of one real variable such that $\lambda(t)=1$ if $t \leqq 1$ and $\lambda(t)=0$ if $t \geqq 1+\delta / 2$, where $\delta>0$ is chosen so small that $\phi$ and $\psi$ extend to diffeomorphisms of $\dot{B}_{1+\delta}^{n}$ into $M$. For $0 \leqq s \leqq 1$ define $F_{s}^{1}$ and $F_{s}^{2}$, maps of $M \rightarrow M$ by

$$
\begin{aligned}
F_{s}^{1}(\phi(t)) & =\phi((1-s \lambda(\|t\|)) t), & & \|t\|<1+\delta, \\
F_{s}^{1}(x) & =x, & & x \in \phi\left(\dot{B}_{1+\delta}^{n}\right), \\
F_{s}^{2}(\psi(t)) & =\psi\left((1-s \lambda(\|t\|))^{-1} t\right), & & \|t\|<1+\delta, \\
F_{s}^{2}(x) & =x, & & x \notin \psi\left(\dot{B}_{1+\delta}^{n}\right) .
\end{aligned}
$$

It is clear that $F_{s}^{1}$ and $F_{s}^{2}$ are diffeomorphisms of $M$ onto itself and that $F_{1-\epsilon}^{2} \circ H \circ F_{1-\epsilon}^{1}=F$ is a diffeomorphism of $M$ onto itself satisfying $\psi=F \circ \phi$. q.e.d.

In [1] we defined the subgroup $G_{0}$ of the group of all diffeomorphisms of $M$ as (roughly) the diffeomorphisms of $M$ which are isotopic to the identity through diffeomorphisms each of which leaves the complement of some compact set fixed. Theorem 5.5 of [1] states that the diffeomorphism $H$ occurring in the proof of Theorem A can be taken in $G_{0}$. Moreover $F_{1-\epsilon}^{1}$ and $F_{1-\epsilon}^{2}$ are also clearly in $G_{0}\left(s \rightarrow F_{s}^{1}\right.$ and $s \rightarrow F_{s}^{2}$ give the desired isotopies) hence

Corollary 1. The $F$ of the above theorem can be taken in $G_{0}$.

Corollary 2. Assume that $M$ is orientable and admits an orientation reversing diffeomorphism onto itself. ${ }^{2}$ Then if $\phi$ and $\psi$ are two differentiable $n$-cells in $M$ there exists a diffeomorphism $F$ of $M$ onto itself such that $\psi=F \circ \phi$.

Proof. By Theorem B we need only consider the case where $\phi$ and $\psi$ define opposite orientations. Let $K$ be an orientation reversing diffeomorphism of $M$ onto itself. Then $K \circ \phi$ is a differentiable $n$-cell in $M$ defining the same orientation as $\psi$, so by Theorem B

${ }^{2}$ This is a nontrivial restriction. If a manifold $M$ has dimension $2 k, \operatorname{dim} H^{k}(M, R)$ $=1$, and $\omega \wedge \omega \neq 0$ for a nonzero element of $H^{k}(M, R)$ then for any homeomorphism $h$ of $M$ onto itself it is clear that $h^{*}(\omega \wedge \omega)=\omega \wedge \omega$ so $h$ is orientation preserving. In particular the complex projective plane is "canonically oriented" in this sense. 
$\psi=H \circ(K \circ \phi)$ for some diffeomorphism $H$ of $M$ onto itself and we put $F=H \circ K$. q.e.d.

\section{Extending diffeomorphisms.}

TheOREM C. Let $\phi$ be a differentiable $k$-cell in $M$ and let $f$ be a diffeomorphism of a neighborhood of $|\phi|$ into $M$ which is assumed to be orientation preserving if $M$ is orientable. Then there exists a diffeomorphism $F$ of $M$ onto itself which agrees with $f$ in a neighborhood of $|\phi|$, and $F$ can even be chosen in $G_{0}$. If $f$ is not orientation preserving then the same conclusion holds (except for $F \in G_{0}$ ) provided $M$ admits at least one orientation reversing diffeomorphism onto itself.

Proof. By Theorem A we can assume $k=n$ and we can also assume that for some $\epsilon>0, \phi$ has been extended to a diffeomorphism of $\dot{B}_{1+2 \epsilon}^{n}$ into the domain of $f$. Let $\phi^{\prime}: B_{1}^{n} \rightarrow M$ be defined by $\phi^{\prime}(t)$ $=\phi((1+\epsilon) t)$. Applying the theorem and corollaries of the preceding section we see that we can find a diffeomorphism $F$ of $M$ onto itself such that $F \circ \phi^{\prime}=f \circ \phi^{\prime}$. Since $\left|\phi^{\prime}\right|$ is a neighborhood of $|\phi|$ this completes the proof.

\section{BIBLIOGRAPHY}

1. R. S. Palais, Natural operations on differential forms, Trans. Amer. Math. Soc. vol. 92 (1959) pp. 125-141.

The Institute for Advanced STUdy 